\title{
Chlamydia awareness - educating high school students about the risks
}

\author{
David G. Allison and Rebecca Lee describe a workshop that was developed and delivered to \\ year 11 high school pupils on chlamydia infections and how to lower the risk of catching it
}

CHLAMYDIA is a sexually transmitted infection (STI) caused by the bacterium Chlamydia trachomatis. The prevalence of infection remains high in the UK and is still rising, with the highest incidence being mainly associated with 16-24 year olds. Of this group, one in 10 is thought to be infected. Moreover, 50 to 80 per cent of all chlamydia infections are asymptomatic, the infection proving successful at remaining undetected in the body for prolonged periods. However, if left undiagnosed and untreated, chlamydia can lead to serious health complications. It is a well established cause of pelvic inflammatory disease, ectopic pregnancy and infertility in females and prostate and testicular problems and infertility in males. Once detected, the infection is fairly easily treated with one dose of antibiotics.

Although chlamydia is a serious and growing public health problem, there are a number of characteristics of this infection which may mean that young people may not consider it threatening. In this respect, pharmacists have the knowledge and opportunity to raise awareness of the significance of chlamydia infections among the general public and to discuss preventive measures which will help reduce infections and associated medical and clinical problems. In addition, pharmacies can also provide easy yet discreet access for young people to sexual health services where they can receive advice, screening and treatment. In order to help circumvent this lack of knowledge, we developed a workshop and delivered it to year 11 high school pupils to encourage strong and realistic perceptions of the threat caused by chlamydia combined with promoting the role of the pharmacist.

\section{About the authors}

David G. Allison is reader in pharmacy education and Rebecca Lee is a final-year MPharm student, both at the School of Pharmacy and Pharmaceutical Science, University of Manchester. Correspondence to David G. Allison (email

david.allison@manchester.ac.uk)

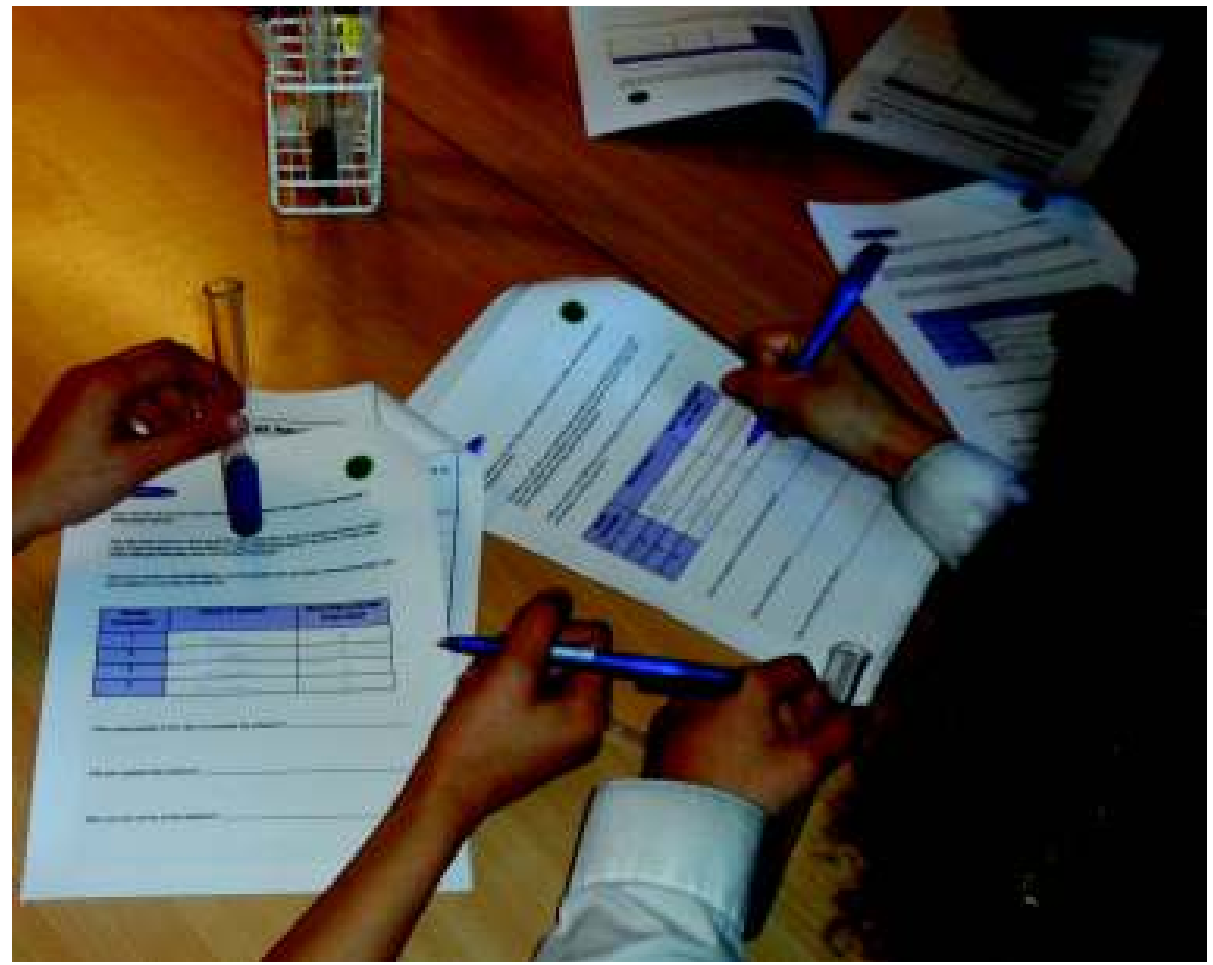

A simulated sexual contact activity (using testwas run among the class to illustrate how easy chlamydia can spread

\section{Spread of infection}

Before delivering the workshop, we visited the high school to distribute a questionnaire to a year 11(GCSE) class in order to gauge their level of knowledge about chlamydia and their preferred learning styles. As a result of this feedback, an additional aim of the workshop was to deliver a mixture of hands-on practical activities and small group discussion to support the Key Stage 4 personal, social and health education curriculum.

The two-hour workshop to 30 pupils representing a cross section of society began with a brief introduction as to who we were and what were going to do. This was followed by a brief Powerpoint presentation describing some of the key features and characteristics associated with chlamydia and other STIs, including some fairly graphic images of reallife infections of male and female genitalia. The aim of this part of the workshop was to get the pupils to identify which genitalia had an infection - easy to spot for syphilis, gonorrhoea and genital warts, not so for chlamydia - thereby reinforcing the asymptomatic nature associated with most chlamydia infections.

Despite the sensitivity of the material being presented, the year 11 pupils responded in a mature manner and, surprisingly, no giggling or embarrassed faces were observed. At this point in proceedings, Helen Edmonds, a community pharmacist from Cohens

Pharmacy, Macclesfield, briefly described the discreet and professional chlamydia screening services that some pharmacies offer. Examples of vulvo-vaginal swabs were distributed among the class prompting comments such as "gosh, it's so small". This was an eye-opener to many as most thought that the swabs would be of a lengthy, cumbersome nature. Further discussions about the pharmacy professional services and use of the swab continued informally throughout the rest of the workshop when the pupils were not actively engaged in a didactic activity. 


\section{Simulated sexual contact activity}

To illustrate how easily the chlamydia bacteria can spread and cause disease, a simulated sexual contact activity was run among the whole class. All but one of the pupils were given a test-tube containing water, while the remaining pupil was given a test-tube containing a starch solution, representing chlamydia. Although the identity of this individual was unknown, care was taken not to give the test-tube to a sensitive or vulnerable individual who might be picked on by the other students after they realised who had been "the carrier". Pupils were required to exchange fluids with four other pupils and make a note in their workbooks whom they had exchanged fluids with and in what order. When all exchanges were complete, each testtube was tested for the presence of

"chlamydia" (starch) by adding a drop of iodine and looking for the characteristic blueblack colour, By tracing back through positive contacts, the original source of infection was identified.

Having established how easily chlamydia infections can be acquired and spread, attempts were made to "infect" a test-tube covered in cling film, with no success, thereby visually demonstrating the effectiveness of condoms as a physical barrier to the spread of infection.

\section{Making the right decision}

Armed with such scientific and pathogenic information about chlamydia, the pupils were then divided into small groups (five or six) and each group given an ethical dilemma to discuss. Each group was required to consider a particular scenario for about 10 minutes, deciding what the key points and arguments were before presenting the case to the rest of the class. Various issues were included in the scenarios, including social pressure, trust, influence of alcohol on sexual behaviour and age discrimination. A common theme with all the scenarios was a reinforcement of the key message that anyone can contract chlamydia at any time from someone who is already infected if they do not use proper precautions. Although some frank and honest discussions occurred within each group of pupils, most were a little shy at presenting their case

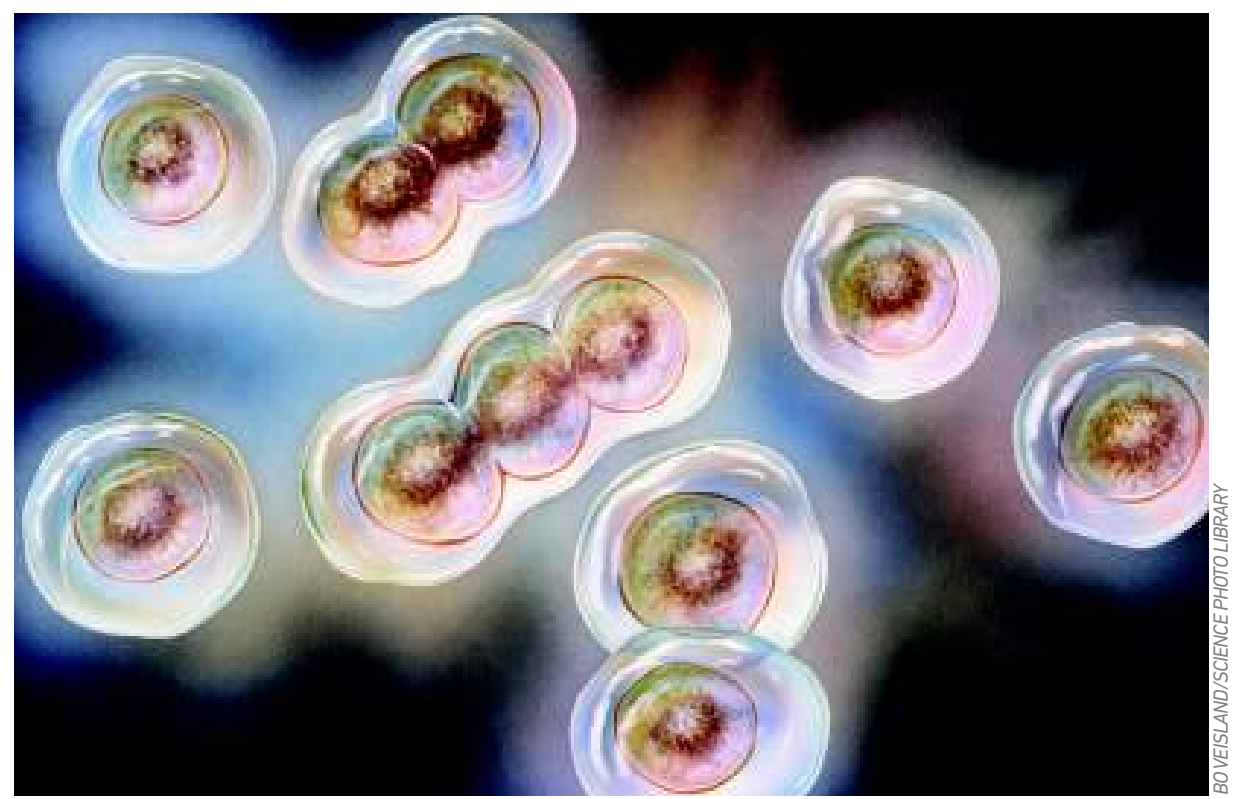

Artwork of chlamydia bacteria: the prevalence of chlamydia infection remains high in the UK and is still rising

in front of their peers. Most, however, had thought through their scenarios thoroughly and offered clear, unambiguous and appropriate advice.

To conclude the workshop, the pupils were asked to design a poster on the control of chlamydia targeted towards 16-24 year olds. Key aspects to incorporate included information on the symptoms and potential outcome of a chlamydia infection, prevention, and where to get tested and treated.

\section{The mission}

The lesson was judged an overwhelming success, with the class teachers and practically all the pupils thoroughly enjoying the activities. We were asked if we could return the following week to present the workshop to another year 11 class, and have been booked for the next academic year. The emphasis on STI prevention in the UK remains fixed on health promotion and education, through improving awareness and encouraging safer sexual behaviour.

As such, the overall aim of this workshop was to raise awareness of chlamydia in young people, in collaboration with primary care sexual health services and through education, presented in a modern context. Considering the method of transmission, the asymptomatic characteristics and the high prevalence of such a disease in a highly specific age range, the importance of education in chlamydia cannot be overstated. Many of the complications require antibiotic therapies and even surgery, which is an unnecessary burden on the NHS and could be reduced by good public awareness either to catch the infection early or through prevention. Pharmacists have an increasing role in public health delivery, and workshops such as this, with little in the way of resource implications, provide an excellent educational vehicle to deliver key and, hopefully, lasting messages.

\section{ACKNOWLEDGEMENTS Perminder}

Banger and the year 11 pupils from Chorlton High School, Manchester, for their help and enthusiastic participation with this activity. Helen Edmonds (Cohens), Syeda Ahmed, Julia Faulkner and Fatima Lunat(second-year pharmacy undergraduate students) for willingly and cheerfully helping out with the workshop delivery. 